\title{
Article
}

\section{Structure and magnetism of orthorhombic epitaxial FeMnAs.}

Dominique Demaille, Gilles Patriarche, Christian Helman, Mahmoud Eddrief, Victor

Hugo Etgens, Maurizio Sacchi, Ana Maria llois, and massimiliano marangolo

Cryst. Growth Des., Just Accepted Manuscript • DOI: 10.1021/cg400576m • Publication Date (Web): 29 Aug 2013

Downloaded from http://pubs.acs.org on September 4, 2013

\section{Just Accepted}

"Just Accepted" manuscripts have been peer-reviewed and accepted for publication. They are posted online prior to technical editing, formatting for publication and author proofing. The American Chemical Society provides "Just Accepted" as a free service to the research community to expedite the dissemination of scientific material as soon as possible after acceptance. "Just Accepted" manuscripts appear in full in PDF format accompanied by an HTML abstract. "Just Accepted" manuscripts have been fully peer reviewed, but should not be considered the official version of record. They are accessible to all readers and citable by the Digital Object Identifier (DOI®). "Just Accepted" is an optional service offered to authors. Therefore, the "Just Accepted" Web site may not include all articles that will be published in the journal. After a manuscript is technically edited and formatted, it will be removed from the "Just Accepted" Web site and published as an ASAP article. Note that technical editing may introduce minor changes to the manuscript text and/or graphics which could affect content, and all legal disclaimers and ethical guidelines that apply to the journal pertain. ACS cannot be held responsible for errors or consequences arising from the use of information contained in these "Just Accepted" manuscripts.

\section{ACS Publications}




\title{
Cover page
}

\section{Structure and magnetism of orthorhombic epitaxial FeMnAs.}

\author{
Dominique Demaille ${ }^{1,2}$, Gilles Patriarche ${ }^{3}$, Christian Helman ${ }^{3,4}$, Mahmoud Eddrief ${ }^{1,2}$, Victor Hugo Etgens ${ }^{1,2}$, \\ Maurizio Sacchi, ${ }^{1,2,5}$, Ana Maria Llois ${ }^{3,4}$ and Massimiliano Marangolo ${ }^{1,2, *}$ \\ ${ }^{1}$ Institut des NanoSciences de Paris, CNRS-UMR7588, Sorbonne Université Pierre et Marie Curie, \\ 4 Place Jussieu, 75005 Paris, France \\ ${ }^{2}$ Laboratorio Internacional Franco-Argentino en Nanociencias (LIFAN) \\ ${ }^{3}$ LPN, CNRS UPR 20, route de Nozay, 91460 Marcoussis, France \\ ${ }^{4}$ CNEA, Centro Atómico Constituyentes, Avenida General Paz 1499, San Martín, Argentina \\ ${ }^{5}$ Synchrotron SOLEIL, L'Orme des merisiers Saint-Aubin, B.P. 48, 91192 Gif-sur-Yvette, France
}

KEYWORDS. FeMnAs, transition metal pnictides, Epitaxy, High Angle Annular Dark Field Scanning Transmission Electron Microscopy (HAADF-STEM), ab initio calculations, magnetic coupling.

\begin{abstract}
The Molecular Beam Epitaxy growth of $\mathrm{Fe}$ on $\mathrm{MnAs} / \mathrm{GaAs}(001)$ leads to the formation of a new nanostructured phase of the FeMnAs compound at the Fe/MnAs interface. We investigate the structural and magnetic properties of this interfacial layer by high angle annular dark field imaging in a scanning transmission electron

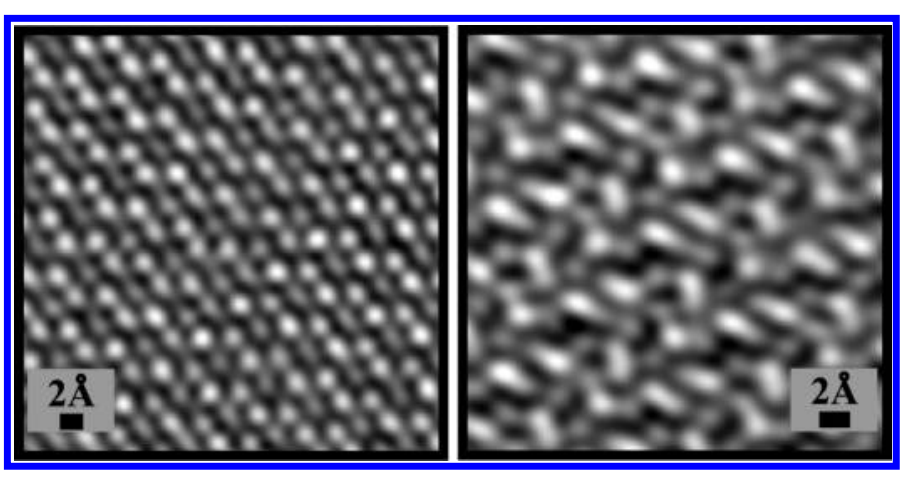
microscope (HAADF-STEM). We determine that the epitaxial FeMnAs layer presents an unusual orthorhombic structure, with vacancy ordering. We completed our study by $a b$ initio calculations and we foresee an antiferromagnetic ground state for this structure.
\end{abstract}

\section{Corresponding Author \\ * Massimiliano Marangolo, marangolo@insp.jussieu.fr}

\section{Author Contributions}

The manuscript was written through contributions of all authors. All authors have given approval to the final version of the manuscript. 


\title{
Structure and magnetism of orthorhombic epitaxial FeMnAs.
}

\author{
Dominique Demaille ${ }^{1,2, *}$, Gilles Patriarche ${ }^{3}$, Christian Helman ${ }^{3,4}$, Mahmoud Eddrief $^{1,2}$, Victor Hugo \\ Etgens $^{1,2}$, Maurizio Sacchi ${ }^{1,2,5}$, Ana Maria Llois ${ }^{3,4}$ and Massimiliano Marangolo $^{1,2}$ \\ ${ }^{1}$ Institut des NanoSciences de Paris, CNRS-UMR7588, Sorbonne Université Pierre et Marie \\ Curie, 4 Place Jussieu, 75005 Paris, France \\ ${ }^{2}$ Laboratorio Internacional Franco-Argentino en Nanociencias (LIFAN) \\ ${ }^{3}$ LPN, CNRS UPR 20, route de Nozay, 91460 Marcoussis, France \\ ${ }^{4}$ CNEA, Centro Atómico Constituyentes, Avenida General Paz 1499, San Martín, Argentina \\ ${ }^{5}$ Synchrotron SOLEIL, L’Orme des merisiers Saint-Aubin, B.P. 48, 91192 Gif-sur-Yvette, \\ France
}

KEYWORDS. FeMnAs, transition metal pnictides, Epitaxy, High Angle Annular Dark Field Scanning Transmission Electron Microscopy (HAADF-STEM), ab initio calculations, magnetic coupling.

\begin{abstract}
The Molecular Beam Epitaxy growth of Fe on MnAs/GaAs(001) leads to the formation of a new nanostructured phase of the FeMnAs compound at the Fe/MnAs interface. We investigate the structural and magnetic properties of this interfacial layer by high angle annular dark field imaging in a scanning transmission electron microscope (HAADF-STEM). We determine that the epitaxial FeMnAs layer presents an unusual orthorhombic structure, with vacancy ordering. We completed our study by ab initio calculations and we foresee an antiferromagnetic ground state for this structure.
\end{abstract}




\section{INTRODUCTION}

Arsenide compounds with composition MM'As, M and M' being 3d transition metals (TM), display a wealth of magnetic and structural phases that depend of the $\mathrm{M} / \mathrm{M}$ ' ratio and on the crystal growth procedure ${ }^{1-3}$. Among them, FeMnAs was found to crystallize in the tetragonal (T) $\mathrm{Fe}_{2} \mathrm{As}$-type structure under normal growth conditions, or in the hexagonal $\mathrm{Fe}_{2} \mathrm{P}$-type $(\mathrm{H})$ one at high pressure and temperature ${ }^{4}$. It was found that the magnetic properties of FeMnAs strongly depend on the crystalline structure: the T phase is antiferromagnetic (AFM) and the H phase is ferromagnetic (FM). The magnetic moments carried by $\mathrm{Mn}$ and Fe depend on the atomic structure, as well ${ }^{4}$.

In this article we report on a new FeMnAs orthorhombic $\left(\mathrm{Co}_{2} \mathrm{P}\right.$-type $)$ phase which is stabilized by epitaxy on a MnAs thin film. MnAs is a ferromagnetic metal that can be epitaxied on $\operatorname{GaAs}(001)$ for spintronics applications ${ }^{5}$. This new FeMnAs phase results from the partial diffusion of Fe during the molecular beam epitaxy (MBE) growth of an iron layer on MnAs. Therefore, we deal with a FeMnAs thin film embedded between pure Fe and MnAs epitaxial films. Here, we address the structural properties of this new phase by High Angle Annular Dark Field (HAADF) imaging in a scanning transmission electron microscope (STEM). This technique provides incoherent images of crystallized materials with atomic resolution. Contrary to the phase contrast High Resolution TEM technique, it allows for a more straightforward interpretation of the sample images, providing direct representations of the atomic columns that can be compared easily to model structures. Finally, by an ab initio study of the electronic and magnetic ground state, we compare the magnetic configurations and the magnetic moment intensities of the new O-phase with the well-known H- and T-phases. Beyond the fundamental interest for the coupling between magnetism and structure in this new phase, two more applicative issues motivated this work, namely Fe/MnAs interdiffusion and colossal magnetocaloric effect (MCE). 
The former is relevant to using Fe/MnAs/GaAs(001) for device applications ${ }^{6-9}$. Here we show that the non-FM ground state of orthorhombic FeMnAs is at the origin of the very low exchangecoupling observed previously in Fe/MnAs hybrid structures, where it was shown that both parallel and anti-parallel magnetic states of Fe and MnAs can be stabilized at low temperature ${ }^{6-7}$. The low magnetic coupling between $\mathrm{Fe}$ and MnAs permits non-inductive ways to store information in magnetic Fe/MnAs hybrid structures ${ }^{9}$.

The latter is important since $\mathrm{Mn}_{1-\mathrm{x}} \mathrm{Fe}_{\mathrm{x}} \mathrm{As}$ is known to exhibit colossal $\mathrm{MCE}$ at room temperature $^{10}$ and at ambient pressure.

Stabilizing MCE in thin films opens up exciting opportunities in micro-scale refrigeration ${ }^{11}$, with the possibility of using high frequency ultrasound triggering ${ }^{12}$ for sensors applications ${ }^{13}$.

\section{EXPERIMENTAL DETAILS.}

The Fe/MnAs/GaAs(001) samples were prepared at the Institut des NanoSciences de Paris by Molecular Beam Epitaxy (MBE), following the procedure described in previous works ${ }^{6}$. First, oxide desorption from the GaAs(001) substrate is carried out under As flux, then a thick GaAs layer is grown under As-rich conditions, with the substrate kept at $T=560{ }^{\circ} \mathrm{C}$. The sample temperature is then reduced to $230{ }^{\circ} \mathrm{C}$ for depositing the $90 \mathrm{~nm}$ thick MnAs layer. The Fe layer is deposited from a Knudsen cell on the template kept at a temperature of $150{ }^{\circ} \mathrm{C}$, where MnAs is single phase and does not display any stripes. Finally, the sample is protected against contamination by $5 \mathrm{~nm}$ of $\mathrm{ZnSe}$.

Previous High Resolution Transmission Electron Microscopy (HRTEM) analyses indicated that Fe grows epitaxially on MnAs with the following relative orientations of the Fe, MnAs and GaAs lattices: $(2-11)_{\mathrm{Fe}} / /(1-100)_{\mathrm{MnAs}} / /(001)_{\mathrm{GaAs}}$ and $[11-1]_{\mathrm{Fe}} / /[001]_{\mathrm{MnAs}} / /[1-10]_{\mathrm{GaAs}}$ (see Fig. 1). It is worth noticing that for few nanometers-thick iron layers, no interdiffused interface layer was clearly evidenced by Trasnsmission Elecron Microscopy studies. Nevertheless, the origin of the weak magnetic exchange coupling between Fe and MnAs was ascribed to the existence of 
an interface reacted layer ${ }^{6}$. Here, we intentionally exaggerated the Fe thickness (around $30 \mathrm{~nm}$ rather than the 'usual' $2-5 \mathrm{~nm}$ ) in order to enhance the reacted region thickness and permit a more comfortable electron microscopy study.

HRTEM and Energy Filtered Transmission Electronic Microscopy (EFTEM) measurements were performed using a Jeol JEM 2100F $200 \mathrm{kV}$ electron microscope equipped with a Gatan GIF electron spectrometer. TEM images evidenced the formation of an intermediate compound between the Fe and MnAs layers. X-ray Energy Dispersive Spectroscopy (XEDS) and EFTEM analysis revealed the presence of $\mathrm{Mn}, \mathrm{Fe}$ and $\mathrm{As}$ in this compound, with average composition FeMnAs.

In order to determine the structure of the FeMnAs compound, we used HAADF-STEM. With a contrast highly sensitive to the atomic number (intensity roughly proportional to $Z^{2}$ ), HAADF-STEM microscopy can distinguish between $\mathrm{Mn}(\mathrm{Z}=25)$ and $\mathrm{As}(\mathrm{Z}=33)$ atoms, but not between $\mathrm{Fe}(\mathrm{Z}=26)$ and $\mathrm{Mn}(\mathrm{Z}=25)$ because of the weak difference of atomic number. HAADF images were obtained on an aberration-corrected STEM (Jeol 2200FS) operating at $200 \mathrm{kV}$. The probe size was $0.1 \mathrm{~nm}(\mathrm{FWHM})$ and the current probe $50 \mathrm{pA}$. The half-angle of convergence of the probe was $30 \mathrm{mrad}$. The half-angle of detection for the dark field (DF) detector was set between $100 \mathrm{mrad}$ (inner) and $170 \mathrm{mrad}$ (outer). Most of the HAADF micrographs were Fourier filtered to improve their quality.

\section{RESULTS AND DISCUSSION}

\section{Along the [100] zone axis of MnAs}

HAADF micrographs were recorded along the [100] MnAs-hexa zone axis; an example is shown in Fig. 2a. An interfacial layer about 10-15 nm wide is clearly evidenced, between the MnAs and the Fe layers. The width of the Fe layer is about $25 \mathrm{~nm}$. One can notice that the interface of the FeMnAs phase is very abrupt with the Fe layer, while it is quite rough with the MnAs layer. The filtered MnAs layer image (Fig. 2b) accurately corresponds to the structure 
expected for MnAs (Fig. 2a lower inset), the brightest spots being ascribed to As atoms and the darkest ones to Mn atoms. This is consistent with the fact that the columns intensity is directly proportional to $\mathrm{Z}^{2}$, since As atoms have high atomic number $\mathrm{Z}$ compared to the $3 \mathrm{~d}$ metal (Mn or Fe) atoms, and no column is composed of mixed elements along this zone axis. The Fe-filtered layer image (Fig. 2d) fits also very well the theoretical Fe structure (Fig. 2a upper inset) projected along the $[011]_{\mathrm{Fe}}$ direction and the intensities of the spots are normally homogeneous, roughly proportional to $\mathrm{Z}_{\mathrm{Fe}}{ }^{2}$. The filtered image of the interfacial layer (Fig. 2c) exhibits a complex structure. Since the growth process of this phase is based on diffusion of iron in the MnAs layer, one could assume that this new compound has hexagonal structure, like MnAs, with tetrahedral and/or octahedral sites partially occupied by iron atoms. A simple comparison with the MnAs phase rules out this interpretation (see Fig. 2b and 2c).

Starting from the idea that our interfacial compound should be an MM'As arsenide, with the additional constraint of a FeMnAs average composition suggested by quantitative analysis of XEDS data, we searched the literature for structural information concerning these compounds. In general, $\mathrm{M}_{2} \mathrm{As}$ arsenides are reported to crystallize in one of the three following structures ${ }^{1}$ : $\mathrm{P}$ $62 \mathrm{~m}$ hexagonal symmetry $\left(\mathrm{Fe}_{2} \mathrm{P}\right.$ structure), Pnma orthorhombic symmetry $\left(\mathrm{Co}_{2} \mathrm{P}\right.$ structure $)$ and $\mathrm{P} 4 / \mathrm{n} \mathrm{mm}$ tetragonal symmetry ( $\mathrm{Fe}_{2} \mathrm{As}$ structure).

According to Ref. 1, As atoms form two types of interstices in the three structures: tetrahedrons and square based pyramids which are alternately stacked along the [001] axis of the hexagonal structure or the [010] direction of the orthorhombic and tetragonal structures (see Fig. 3). One can therefore define a rhombic pseudo unit cell built with one tetrahedron and one square pyramid, both sharing a triangular face. The difference between the 3 types of structure lies in the ordering of these rhombic blocks. The metal atoms are always located in the tetrahedral and square based pyramidal sites ${ }^{1-3}$.

Most of the binary phases $\mathrm{M}_{2} \mathrm{As}$ have a tetragonal structure $\left(\mathrm{Fe}_{2} \mathrm{As}, \mathrm{Mn}_{2} \mathrm{As}, \mathrm{Cr}_{2} \mathrm{As}\right)$ while ternary phases MM'As have hexagonal symmetry, except FeMnAs whose structure is 
tetragonal under normal growth conditions ${ }^{1-2}$. We didn't find any TEM study on these materials, but the STEM-HAADF technique, as we previously pointed out, allowed us to compare directly the HAADF micrographs with the theoretical structures of $\mathrm{Fe}_{2} \mathrm{As}, \mathrm{Fe}_{2} \mathrm{P}, \mathrm{Co}_{2} \mathrm{P}$.

The image corresponding to our interfacial compound was compared to the three known structures of the arsenides depicted along the [001] direction for $\mathrm{Fe}_{2} \mathrm{P}$ and along the [010] direction for $\mathrm{Fe}_{2} \mathrm{As}$ and $\mathrm{Co}_{2} \mathrm{P}$ in the publication of $\mathrm{A}$ Nylund et al. ${ }^{1}$ (Fig. 4a-c). An accurate coincidence of the atomic columns of the interfacial FeMnAs compound (Fig. 4d) with the atomic positions of the $\mathrm{Co}_{2} \mathrm{P}$ type structure was evidenced if $\mathrm{P}$ is replaced by As and Co by $\mathrm{Mn}$ or Fe. To facilitate the comparison several markers have been drawn on the HAADF micrograph, using the following convention: big yellow circles show the periodicity of the pattern, green and blue filled circles represent the pyramidal and tetrahedral sites occupied by the metal $(\mathrm{M}=\mathrm{Mn}$ or Fe), respectively, pink and red filled circles indicate the As columns, pink lines point out the rhombic blocks (pyramid + tetrahedron) and the blue dotted lines delimit the unit cell. The agreement is also good for the intensities which are approximately proportional to $\mathrm{Z}_{\mathrm{As}}{ }^{2}$ for the white spots and to $\mathrm{Z}_{\mathrm{M}}{ }^{2}(\mathrm{M}=\mathrm{Mn}, \mathrm{Fe})$ for the grey spots.

Therefore, the resulting relationship with the MnAs structure would be $[100]_{\text {MnAs-hexa }} / /$ $[010]_{(\mathrm{Fe}, \mathrm{Mn}) \text { As-ortho }},[001]_{\text {MnAs-hexa }} / /[100]_{(\mathrm{Fe}, \mathrm{Mn}) \text { As-ortho. The parameters }} \mathrm{a}_{\text {ortho }}=6.36 \AA$ and $\mathrm{c}_{\text {ortho }}=$ $7.04 \AA$ have been obtained from the HAADF images along the $[010]_{(\mathrm{Fe}, \mathrm{Mn}) \text { As-ortho. The }} \mathrm{b}_{\text {ortho }}$ parameter was measured on HAADF images along the $[100]_{(\mathrm{Fe}, \mathrm{Mn}) \text { As-ortho }}$ perpendicular zone axis. All the experimental values are given in Tab.1. Several projected distances were thus calculated from the theoretical $\mathrm{Co}_{2} \mathrm{P}$ type structure with these parameters. They agree with the measured distances between the atomic columns displayed on Fig. 4d. The calculated projected distances between the As columns and the closest metal columns are $1.48 \AA, 1.83 \AA$ and $2.0 \AA$. The measured values (respectively 1.4 $\AA, 1.9 \AA$ and $2.0 \AA$ ) are in good agreement with these calculated values. Let us draw the attention to the fact that these values are close to the 
microscope spatial resolution ( $1 \AA$ ), which may explain why the As and M columns are not always well resolved.

This meticulous observation along the $[100]_{\text {MnAs-hexa }}$ direction supports the validity of the $\mathrm{Co}_{2} \mathrm{P}$ type structure. In the discussion that follows, we assume that our FeMnAs interfacial layer has orthorhombic $\mathrm{Co}_{2} \mathrm{P}$-type structure, showing that all TEM results are coherent with this hypothesis. We will refer to this orthorhombic structure O-FeMnAs.

\section{Diffraction patterns along $[\mathbf{1 0 0}]_{\text {MnAs-hexa }} / /[010]_{(\mathrm{Fe}, \mathrm{Mn}) \text { As-ortho }}$ and $[001]_{\text {MnAs-hexa }} / /$} $[100]_{(\mathrm{Fe}, M n) A s-o r t h o}$

Diffraction measurements represent a suitable way to confirm the FeMnAs orthorhombic structure. Let us recall some information about the Pnma space group. The existence of a diagonal glide plane ' $\mathrm{n}$ ' $\perp$ a and an axial glide plane 'a' $\perp \mathrm{c}$ implies the reflection conditions $0 \mathrm{kl}$ $: \mathrm{k}+\mathrm{l}=2 \mathrm{n}$ and $\mathrm{hk} 0: \mathrm{h}=2 \mathrm{n}$ respectively.

The Fast Fourier Transform (FFT) performed on our FeMnAs-O phase along its [010] zone axis (Fig. 5a) shows a nearly square lattice with $7.1 \AA$ and $6.4 \AA$ interplanar distances values, matching very well to the $\mathrm{d}_{001}=7.04 \AA$ and $\mathrm{d}_{100}=6.36 \AA$ of the orthorhombic structure, whose reciprocal lattice is presented in Fig. 5b. One must specify that the normally forbidden h00: $\mathrm{h}=2 \mathrm{n}+1$ and $001: \mathrm{l}=2 \mathrm{n}+1$ reflexions are excited by the dynamical effects of the electronic diffraction.

To complete the study, we analyze the diffraction micrograph along the [100] zone axis of our FeMnAs-O layer. Despite appearances, Fig. 6a does not depict a hexagonal symmetry but an orthorhombic symmetry with extinctions. The zone axis is not six-fold, like for MnAs; four angles were measured to be approximately $62^{\circ}$ whereas the two others were close to $57^{\circ}$. This diffraction pattern fits exactly the reciprocal lattice of the orthorhombic structure along the [100] direction (Fig. 6b), characterized by four angles equal to $61.48^{\circ}$ [between $(002)$ and $(011),(002)$ and (0-11)], two angles equal to $57.03^{\circ}$ [between (011) and (01-1)] and measured values in 8 
agreement with $\mathrm{d}_{011}=3.36 \AA$ and $\mathrm{d}_{002}=3.52 \AA$. We must point out that the spots corresponding to the (011) and (002) plane families, close to the centre of the diffraction pattern, are not visible because they merge with the MnAs spots. We can conclude that the diffraction results confirm unequivocally the orthorhombic structure of our FeMnAs phase, with the lattice parameters given in the previous section.

\section{Along the [001] zone axis of MnAs : a compound with arranged vacancies}

A series of HAADF images along the $[001]_{\text {MnAs-hexa }}$ zone axis were also recorded; an example is shown in Fig. 7a. Similar comments as those made on Fig. 2a apply, in particular concerning the exact coincidence between the MnAs and Fe layers with their theoretical structures and the difference of roughness for the two interfaces MnAs-FeMnAs and FeMnAsFe. But, contrary to the Fig. 2c, the O-FeMnAs layer picture exhibits areas with different intensity distributions (Fig. 7c). This will be discussed further in the rest of the paper. If we first consider the [001] oriented MnAs layer, the HAADF micrograph corresponds perfectly well to the projected MnAs structure. We notice that all the columns of the HAADF image (Fig. 7b) have the same intensity, although they are supposed to be alternately As and Mn columns (Fig. 7a, upper inset). The MnAs cell has two atomic positions for Mn (0,0,0 and 0,0,1/2) which are aligned along the c-axis direction, whereas the two As positions (1/3, 2/3, $1 / 4$ and 2/3, 1/3, 3/4) are not superposed along this axis. Therefore, Mn columns are twice as dense (intensity proportional to $2 \mathrm{Z}_{\mathrm{Mn}^{2}}{ }^{2}$ ) as the As columns (intensity proportional to $\mathrm{Z}_{\mathrm{As}}{ }^{2}$ ). Since $2 \mathrm{Z}_{\mathrm{Mn}}{ }^{2}$ is nearly equal to $\mathrm{Z}_{\mathrm{As}}{ }^{2}$, the intensities are not much different. Also the filtered Fe layer image (Fig. 7d) coincides well, for positions and intensities, with the theoretical Fe structure projected along the $[11-1]_{\mathrm{Fe}}$ direction (Fig. 7a, lower inset).

From these HAADF images of the O-FeMnAs layer along the $[100]_{(\mathrm{Fe}, \mathrm{Mn}) \text { As-ortho, }}$ the $\mathrm{b}_{\text {ortho }}$ parameter was calculated to be $3.825 \AA$. Taking into account the three lattice parameters measured from the HAADF images, the O-FeMnAs structure along the [100] direction is 
reported in Fig. 8b. In this figure, the red filled circles represent the As atoms while the green and blue circles represent respectively the pyramidal and tetrahedral sites occupied by the metal $(\mathrm{M}=\mathrm{Mn}$ or $\mathrm{Fe}$ ). The O-FeMnAs structure is compared to the filtered HAADF image shown in Fig. 8a (zone 1). If we consider the As columns of the theoretical structure, we can see a coincidence with the brightest spots of the HAADF micrograph. The obvious difference is the systematic existence of the columns corresponding to the tetrahedral metal sites (blue filled circles in Fig. 8b) very close to the As columns in the schematic structure which are not evidenced in the micrograph. The projected distance $(\mathrm{d}=0.42 \AA)$ between these As and M columns, calculated from the theoretical structure, is smaller than the microscope resolution (1 $\AA$ ), which explains why we see only one spot, whose intensity should be roughly proportional to $\mathrm{Z}_{\mathrm{As}}{ }^{2}+\mathrm{Z}_{\mathrm{M}}{ }^{2}$. If we take the average position (along the 'c' axis) of these very close As and $\mathrm{M}$ columns, the calculated distances $\left(\mathrm{d}_{(5,6-7,8)}=, \mathrm{d}(7,8-11,12)=2.185 \AA, \mathrm{d}(5,6-13,14)=4.86 \AA\right.$ in Fig. $\left.8 \mathrm{~b}\right)$ match very well the measured distance between the bright spots in Fig. $8 \mathrm{a}(\mathrm{d} 1=, \mathrm{d} 2=2.3 \AA$, $\mathrm{d} 3=5.0 \AA)$.

When examining the upper part of the schematic structure of Fig. $8 \mathrm{~b}$ (along the ' $a$ ' axis), one expects that two levels of intensity in the HAADF micrograph: the higher proportional to $\mathrm{Z}_{\mathrm{As}}{ }^{2}+$ $\mathrm{Z}_{\mathrm{M}}{ }^{2}$ for the pairs of As and $\mathrm{M}$ columns mentioned above (red empty circles in Fig. 8b), the lower proportional to $\mathrm{Z}_{\mathrm{M}}^{2}$ for the pyramidal metal sites (green filled circles).

The pairs of As and $\mathrm{M}$ columns make a 2D pseudo-hexagonal network (highlighted by red lines in the Fig. 8a,b), delimiting an area that contains two atomic columns which correspond to the pyramidal metal sites of much lower intensity.

Arrows in Fig. 8a,b indicate spots where vacancies have replaced the second metal column.

In some parts of the micrograph (Fig. 8a, zone 2), the spot intensity distribution is different, with three intensity levels. The brightest spots (proportional to $\mathrm{Z}_{\mathrm{As}}{ }^{2}+\mathrm{Z}_{\mathrm{M}}{ }^{2}$ ) form a pseudotriangular network (red drawing in Fig. 8a,b), which can be interpreted as two out of the four tetrahedral sites being vacant (represented by a cross in Fig. 8b). The spots surrounded by 
these 'triangles' are alternately grey and white, meaning that two of the four pyramidal sites are vacant as it is schematized at the bottom of Fig. 8b. Consequently, spots surrounded by these pseudo-triangles have alternatively an intensity proportional to $\mathrm{Z}_{\mathrm{M}}{ }^{2}$ (grey spot) and to $\mathrm{Z}_{\mathrm{As}}{ }^{2}$ (white spot).

The interpretation of the intensities in a HAADF micrograph of such a complex structure is not an easy task, and no quantitative investigation has been attempted. Nonetheless, we can draw a qualitative picture from our analysis, showing that the structure of the interfacial FeMnAs phase is composed of As pyramids and tetrahedra, forming the frame of the structure, containing metal atoms (Mn or Fe) or vacancies. The presence, in some areas of the sample, of an ordered vacancy network may correspond to local composition variations of the O-FeMnAs phase. Our results are at variance with published data on bulk FeMnAs, reporting crystallization in the $\mathrm{Fe}_{2} \mathrm{As}$ tetragonal or hexagonal symmetry and not mentioning the existence of vacancies in the structure. Both the unusual structure and the presence of vacancies have to be related to the way we formed the FeMnAs sample, i.e. diffusion of iron in the MnAs layer during growth, under the action of epitaxial constraints.

It was shown, by neutron diffraction, that Fe and Mn atoms in both tetragonal and hexagonal MnFeAs bulk phases are located at tetrahedral and pyramidal sites respectively ${ }^{4,14}$. We couldn't prove that such a Mn and Fe crystallographic ordering exists in our FeMnAs layer because, as we said previously, STEM-HAADF technique cannot distinguish between Fe and Mn.

\section{Magnetism of orthorhombic FeMnAs.}

In the following we address the magnetic properties of the unusual orthorhombic (O) structure of FeMnAs. As mentioned above, FeMnAs crystallizes "naturally" in the tetragonal (T) structure and, under pressure, in the hexagonal $(\mathrm{H})$ one. It has been shown that the T-structure presents a larger volume and an antiferromagnetic (AFM) ground state with a Néel temperature of $463 \mathrm{~K}$. The H-phase is ferromagnetic (FM) with a Curie temperature near to $190 \mathrm{~K}$ (see 
Ref. 4 and references therein]. It has also been reported that the magnetic moments carried by the $\mathrm{Mn}$ and Fe atoms strongly depend on the atomic structure and particularly of the metal to metal (M-M) distance, $\mathrm{d}_{\mathrm{MM}}$. It turns out that the larger is $\mathrm{d}_{\mathrm{MM}}$ the more intense will be the magnetic moment of $\mathrm{Fe}$ and $\mathrm{Mn}^{4}$. This empirical observation makes us to expect that the observed orthorhombic structure that presents an enhanced cell-volume $(+7 \%$ and $+2 \%$ than the $\mathrm{H}$ and $\mathrm{T}$ phase, respectively) will present an AFM ground state with an increased magnetic moment for the $\mathrm{Mn}$ and $\mathrm{Fe}$ atoms.

We performed electronic calculations using $a b$ initio density functional theory as implemented in the VASP code ${ }^{15}$. Projector Augmented Wave (PAW) pseudopotentials are used $^{16}$, together with the generalized gradient approximation as parameterized by Perdew et al. ${ }^{17}$ for the exchange and correlation potential. We consider a plane wave basis set with kinetic energy values up to $337 \mathrm{eV}$ and $432 \mathrm{k}$-points in the irreducible Brillouin zone. We found that the cell parameters agree with experimental values within $3 \%$ of confidence.

A comparison of magnetic configurations and calculated magnetic moments is given in table I. In order to perform a fruitful comparison with values reported in the literature ${ }^{4,14,18}$ we performed $a b$ initio calculations for the $\mathrm{H}, \mathrm{T}$ and O-FeMnAs phases. Our results reproduce closely the ones reported in references 4 and 18 for $\mathrm{H}$ and reference 14 for T-FeMnAs. Despite the overall agreement, we report that the value of the magnetic moment of Fe in the tetragonal position of the $\mathrm{T}$ phase is higher than the value obtained by Tobola et al. ${ }^{4}$ Nevertheless, it agrees well with the range of values reported by other authors even using the same calculation method $^{19}$. Similarly to what happens in $\mathrm{H}$ and $\mathrm{T}$ phases ${ }^{4}$, we confirm the localization of Fe and Mn on tetrahedral and pyramidal sites, respectively.

Below, we focus on the main magnetic properties that come out from our calculations.

The major points concerning the O-phase are the following:

The orthorhombic cell presents an AFM ground state similarly to the tetragonal one (tab.1). In order to find the magnetic ground state, we tested several magnetic configurations. Having in 
mind that the arrangement of the atoms along c-axis is $\mathrm{t} p \mathrm{ptt} p \mathrm{pt}$, where ' $\mathrm{t}$ ' and ' $\mathrm{p}$ ' mean the tetrahedron and square pyramid sites respectively (see Fig. 3), the magnetic configuration obtained is $+-+\ldots+++$. This indicates that t-type positions (occupied by Fe atoms) prefer a ferromagnetic alignment, while p-type (Mn atoms) prefer an antiferromagnetic orientation. The antiferromagnetic alignment of the p-type positions is consistent with what happened in tetragonal and hexagonal structures ${ }^{4}$.

The Fe and Mn magnetic moments increase significantly as compared to the values reported in $\mathrm{H}$ and $\mathrm{T}$ phases. In particular the one computed for $\mathrm{Fe}$ is equal to $1.6 \mu_{\mathrm{B}}$.

In order to estimate the magnetic coupling between $\mathrm{Fe}$ and $\mathrm{Mn}$ we calculated the energy difference between ground state and a second magnetic configuration. This second magnetic configuration is similar to the magnetic ground state but with all Mn magnetic moment within our unit cell inverted. The obtained energy difference value of $6 \mathrm{meV}$ per magnetic ion reveals a weak magnetic coupling between Fe-Mn leading to an antiferromagnetic configuration of the magnetic moments of the unit cell.

\section{CONCLUSION}

We have shown, by STEM-HAADF microscopy measurements, the existence of a new structural phase of FeMnAs stabilized by the epitaxy of Fe on MnAs/GaAs(001). A FeMnAs layer forms at the growth interface, and adopts an orthorhombic structure. This new phase presents a large unit cell volume, if compared to the most known hexagonal and tetragonal bulk phases. Interestingly, we found ordered vacancies with composition variations suggesting that the orthorhombic FeMnAs phase is not stoichiometric. In order to give an insight into the magnetic properties of this system we performed $a b$ initio calculations that indicate that the ground state of this new FeMnAs phase is antiferromagnetic. 
FIGURES

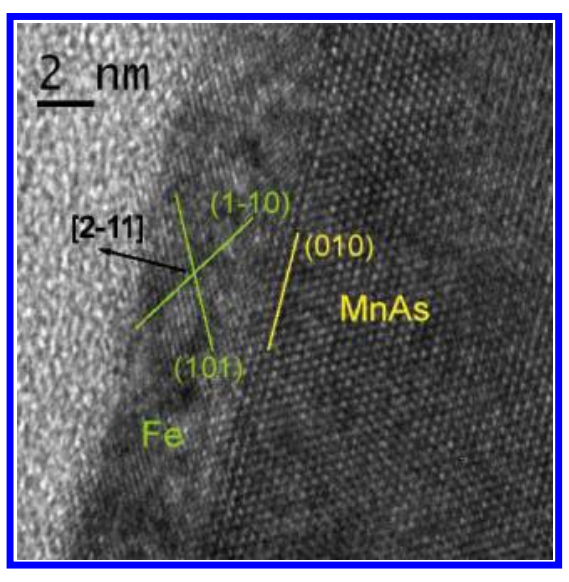

Figure 1. HRTEM cross-

sectional image of

$\mathrm{Fe} / \mathrm{MnAs} / \mathrm{GaAs}(001)$ along c

axis of MnAs for a thin $\mathrm{Fe}$

layer. 


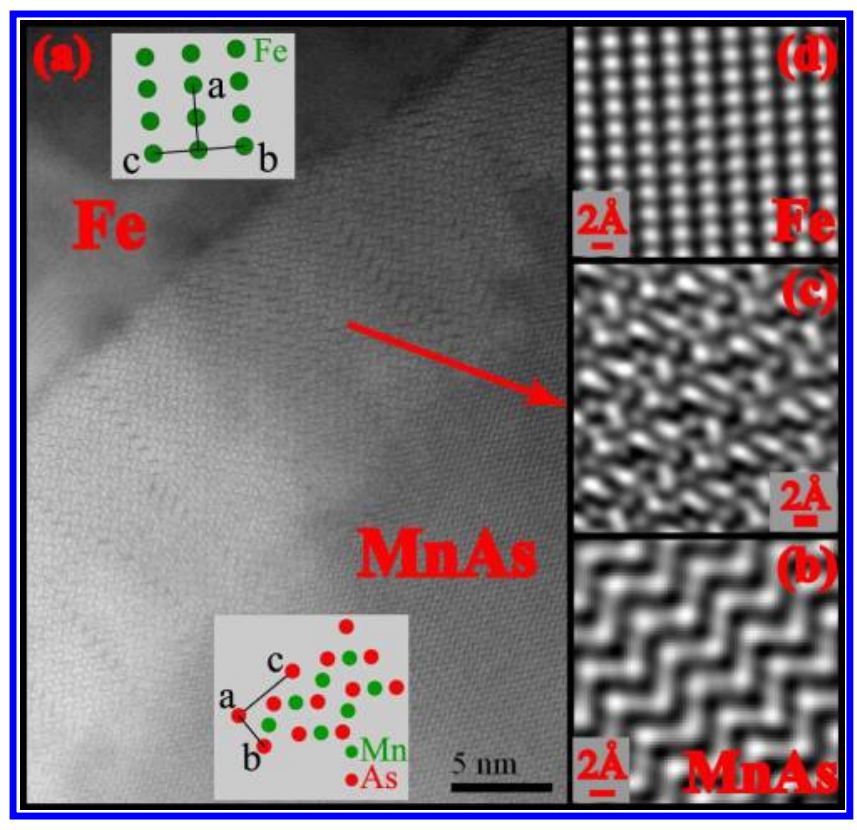

Figure 2. (a) STEM-HAADF cross-sectional image of $\mathrm{Fe} / \mathrm{MnAs} / \mathrm{GaAs}(001)$ along [100] axis of MnAs for a thick Fe layer. Filtered images of (b) MnAs layer (c) intermediate compound (d) Fe layer. 


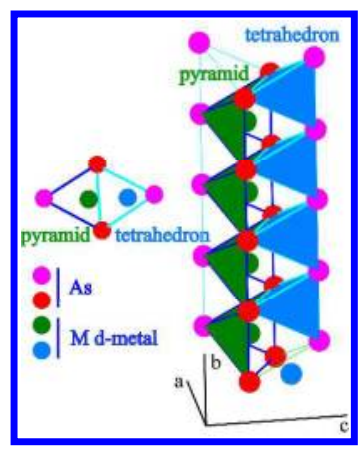

Figure 3.

Tetrahedrons and

square based c

pyramids alternately

stacked along the

[001] axis of the

hexagonal structure

or the [010]

direction of the

orthorhombic and

tetragonal

structures. 


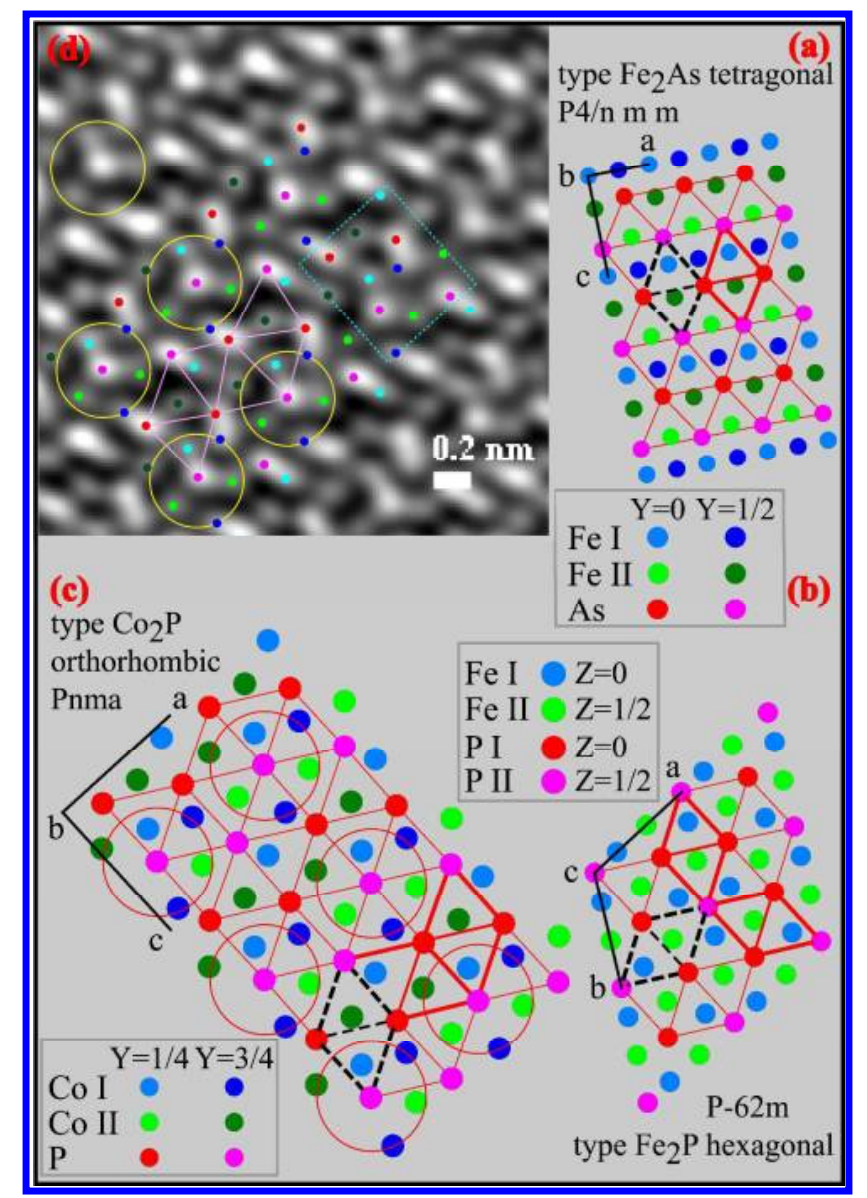

Figure 4. Schematic structure of (a) Tetragonal

$\mathrm{Fe}_{2} \mathrm{As}$ along [010] direction (b) Hexagonal $\mathrm{Fe}_{2} \mathrm{P}$ along [001] direction (c) Orthorhombic Co2P along [010] direction. (d) Filtered STEMHAADF cross-sectional image of the intermediate compound. 


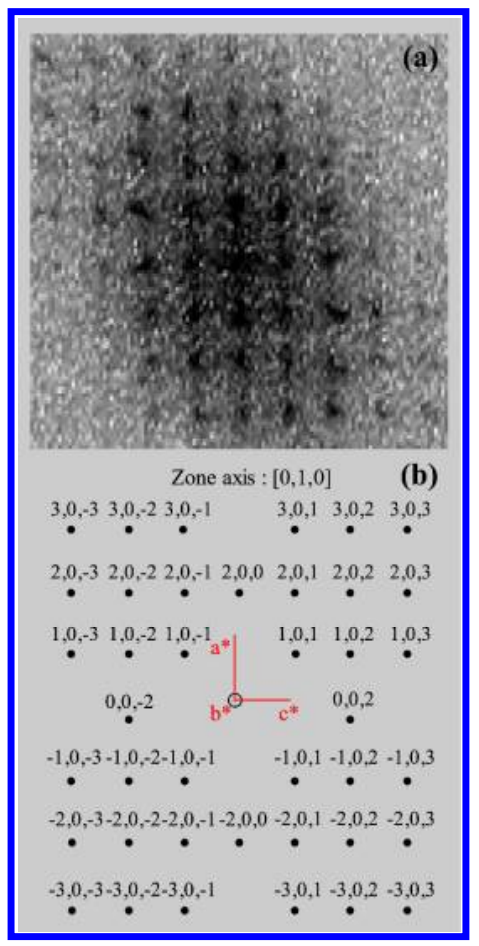

Figure 5. (a) Fast Fourier

transform (FFT) obtained

from the image of the

intermediate compound

(along the [100] zone axis

of MnAs). (b) Reciprocal

lattice of the

orthorhombic Co2P type

structure along its [010]

zone axis. 


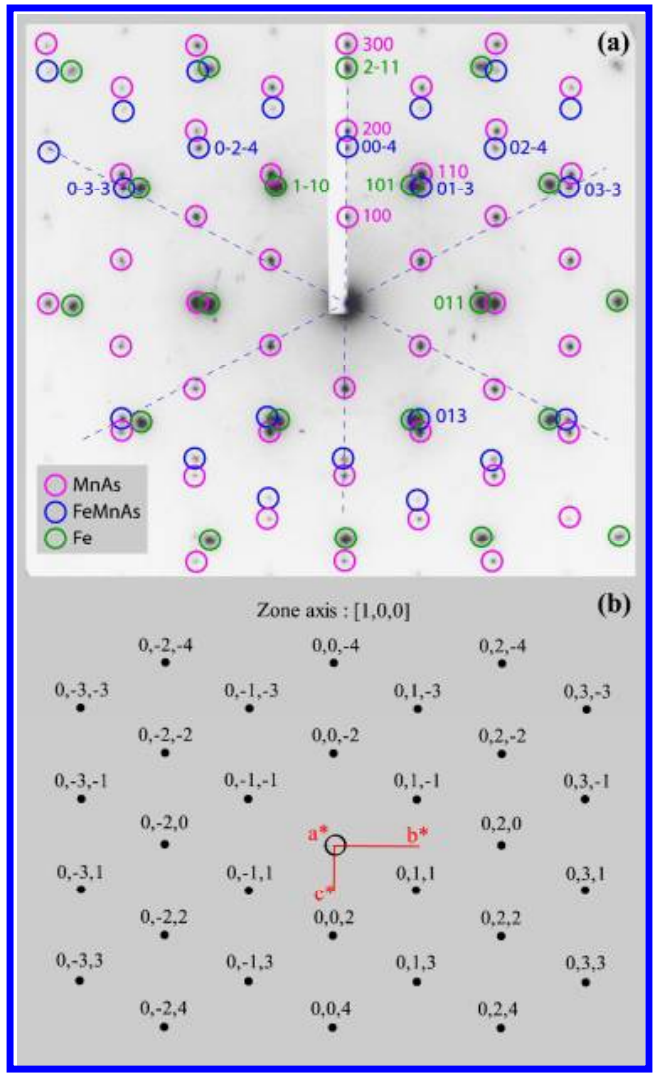

Figure 6. (a) Diffraction pattern obtained for $\mathrm{Fe} / \mathrm{MnAs} / \mathrm{GaAs}(001)$ sample along the [001] zone axis of MnAs. Spots indicated by blue squares are those of the intermediate compound. (b) Reciprocal lattice of the orthorhombic Co2P type structure along its [100] zone axis. 


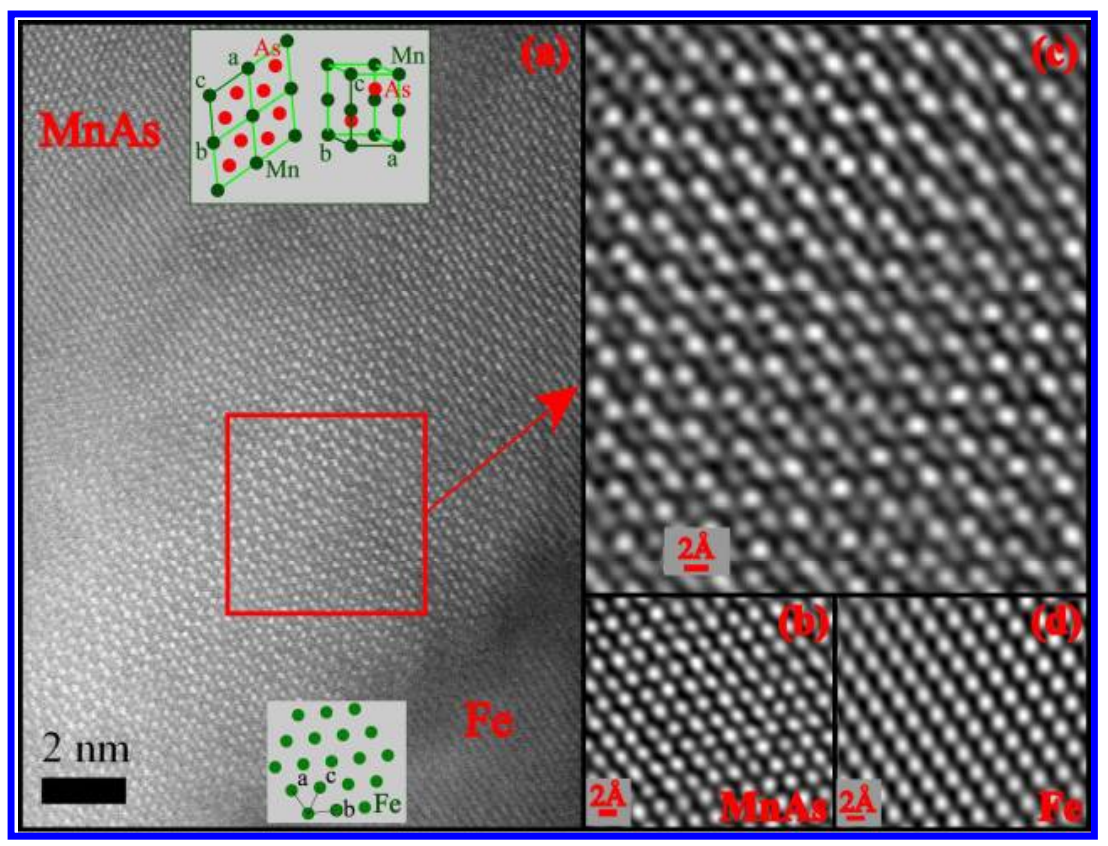

Figure 7. (a) STEM-HAADF cross-sectional image of Fe/MnAs/GaAs(001) along [001] axis of MnAs for a thick Fe layer. Filtered images of (b) MnAs layer (c) intermediate compound (d) Fe layer. 


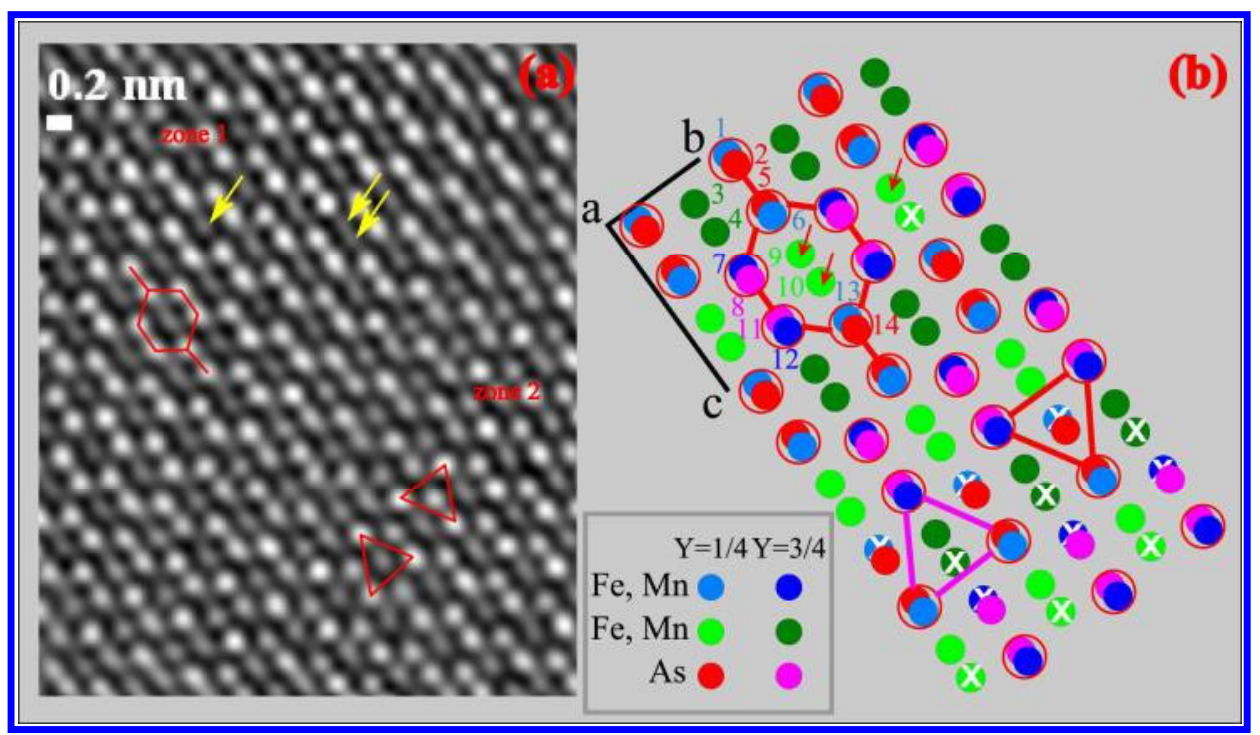

Figure 8. (a) Filtered HAADF image of the intermediate compound. (b) Schematic structure of orthorhombic Pnma 'FeMnAs' along its [100] zone axis with vacancies (represented by white crosses). Green and blue filled circles correspond respectively to the pyramidal and tetrahedral sites occupied by the metal atoms. White crosses symbolize the vacancies in the structure. 
Structural and magnetic parameters.

\begin{tabular}{|c|c|c|c|}
\hline $\begin{array}{l}\text { Structural } \\
\text { phase }\end{array}$ & $\mathrm{H}$ & $\mathrm{T}$ & $\mathrm{O}$ \\
\hline $\mathrm{a}(\AA)$ & 6.26 & 3.74 & 6.36 \\
\hline $\mathrm{b}(\AA)$ & & & 3.825 \\
\hline c $(\AA)$ & 3.54 & 5.99 & 7.04 \\
\hline $\begin{array}{l}\text { Volume / Z } \\
\left(\AA^{3}\right)^{*}\end{array}$ & 13.38 & 14.00 & 14.27 \\
\hline $\begin{array}{l}\text { Magnetic } \\
\text { configuration }\end{array}$ & $\mathrm{F}$ & $\mathrm{AF}$ & $\mathrm{AF}$ \\
\hline $\mathrm{Fe}(\mathrm{t}-$ site $)\left(\mu_{\mathrm{B}}\right)$ & $\begin{array}{c}1.2 \\
(1.1)\end{array}$ & $\begin{array}{l}0.6 * * \\
(0.03)\end{array}$ & 1.6 \\
\hline $\begin{array}{l}\text { Mn (p-site) } \\
\left(\mu_{B}\right)\end{array}$ & $\begin{array}{c}3.11 \\
(3.13)\end{array}$ & $\begin{array}{c}3.30 \\
(3.36)\end{array}$ & 3.44 \\
\hline
\end{tabular}

Table 1. Experimental lattice parameters obtained for the hexagonal, tetragonal (values from Ref. 3) and orthorhombic (our measurements) structures. Magnetic ground-state-configuration and magnetic moments obtained by our ab initio calculations. In brackets similar results by Tobola et $\mathrm{all}^{3}$. * $\mathrm{Z}$ is the number of formula units in the unit cell. Volume is divided by $\mathrm{Z}$ to facilitate the comparison between the 3 structures. ** In agreement with calculations of Ref 19.

\section{AUTHOR INFORMATION}

\section{Corresponding Author}

* Dominique Demaille, demaille@insp.jussieu.fr

\section{Author Contributions}

The manuscript was written through contributions of all authors. All authors have given approval to the final version of the manuscript.

\section{REFERENCES}

[1] Nylund, A.; Roger, A.; Sénateur, J.P.; Fruchart, R. J. of Solid State Chemistry 1972, 4, 115 
[2] Fruchart, D. Solid State Sciences 2005, 7, 767

[3] Sénateur J.P.; Fruchart D.; Boursier, D.; Rouault, A. Colloque C7, supplément au nº 12 1997, Tome 38, page C7

[4] Tobola, J.; Bacmann, M.; Fruchart, D.; Wolfers, P.; Kaprzyk, S.; Koumina, A.A. J. of Alloys and Compounds 2001, 317-318 274-279

[5] Garcia V; Jaffres H; Eddrief M; Marangolo, M. ; Etgens, V.H.; George J.M. Phys. Rev. B. $\mathbf{2 0 0 5}, 72,081303$

[6] Sacchi, M.; Marangolo, M.; Spezzani, C.; Coelho, L.; Breitwieser, R.; Milano, J.; Etgens, V. H. Phys. Rev. B 2008, 77, 165317

[7] « Procédé de modification de la direction d'aimantation d'une couche ferromagnétique » Demande internationale PCT/FR2010/051274

[8] Sacchi, M.; Marangolo, M.; Spezzani, C.; Breitwieser, R.; Popescu, H.; Dealaunay, R.; Salles, B. R.; Eddrief, M.; Etgens, V. H. Phys.Rev.B 2010, 81, 220401(R)

[9] Helman, C.; Milano, J.; Tacchi, S.; Madami, M.; Carlotti, G.; Gubbiotti, G.; Alejandro, G.; Marangolo, M.; Demaille, D.; Etgens, V. H.; Pini M. G. Phys.Rev.B 2010, 82, 094423

[10] de Campos, A.; Rocco, D.L.; Carvalho, A.M.G.; Caron, L.; Coelho, A.A.; Gama, S.; Da Silva, L.M.; Gandra, F.C.G.; Dos Santos, A.O.; Cardoso, L.P.; Von Ranke, P. J. and De Oliveira, N.A. Nature Materials 2006, Vol 5, 802

[11] Mosca, D.H.; Vidal, F.; Etgens, V. H. Physical Review Letters 2008, 101, 125503

[12] Duquesne, J. Y.; Prieur, J. Y.; Agudo Canalejo, J.; Etgens, V. H.; Eddrief, M.; Ferreira, A.L.; Marangolo, M. Physical Review B 2012, 86, 035207 
[13] « Dispositif Piézoélectrique » Demande internationale PCT/ FR 1155988

[14] Baron, V.; Neronin, N.; Rundlof, H.; Tellgren, R. J. of Magn. And Magn. Mat. 1997, 169271

[15] Kresse, G. and Furthmller, J. Computational Materials Science 1996, vol. 6, no. 1, 15.

[16] Kresse, G. and Joubert, D. Phvs. Rev. B 1999, vol. 59, 1758.

[17] Perdew, J. P.; Burke, K.; and Ernzerhof, M. Phvs.Rev.Lett. 1996, vol. 77, 3865.

[18] Liu, X.B.; Altounian, Z. 2009 J. of Magnetism and Magnetic Materials 2009, 3212005.

[19] Val'kov, V. I. and Golovchan, A. V. Low-Temperature phvsics 2008, 34, 43. 
For Table of Contents Use Only

\section{Structure and magnetism of orthorhombic epitaxial FeMnAs.}

Dominique Demaille*, Gilles Patriarche, Christian Helman, Mahmoud Eddrief, Victor Hugo Etgens, Maurizio Sacchi, Ana Maria Llois and Massimiliano Marangolo.

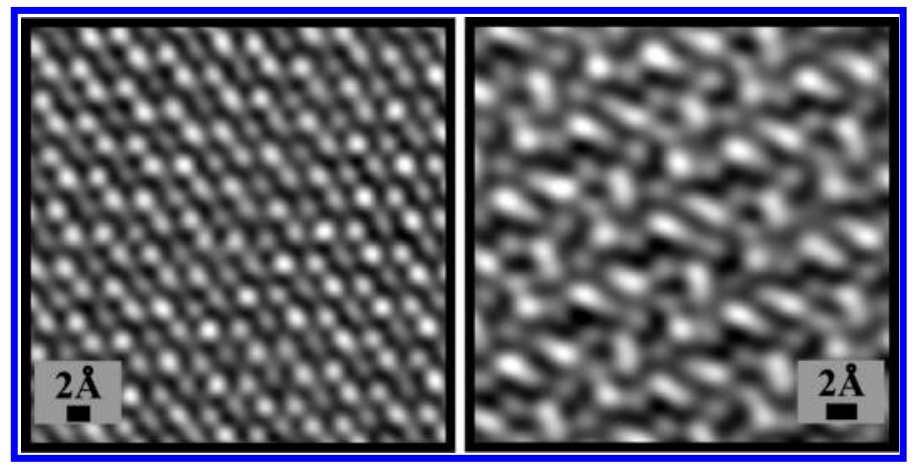

Synopsis: The Molecular Beam Epitaxy growth of Fe on MnAs/GaAs(001) leads to the formation of an epitaxial FeMnAs phase at the Fe/MnAs interface. The investigation of the structure by High Angle Annular Dark Field imaging in a Scanning Transmission Electron Microscope reveals an unusual orthorhombic structure, with vacancy ordering. Ab initio calculations show an antiferromagnetic ground state for this orthorhombic FeMnAs. 


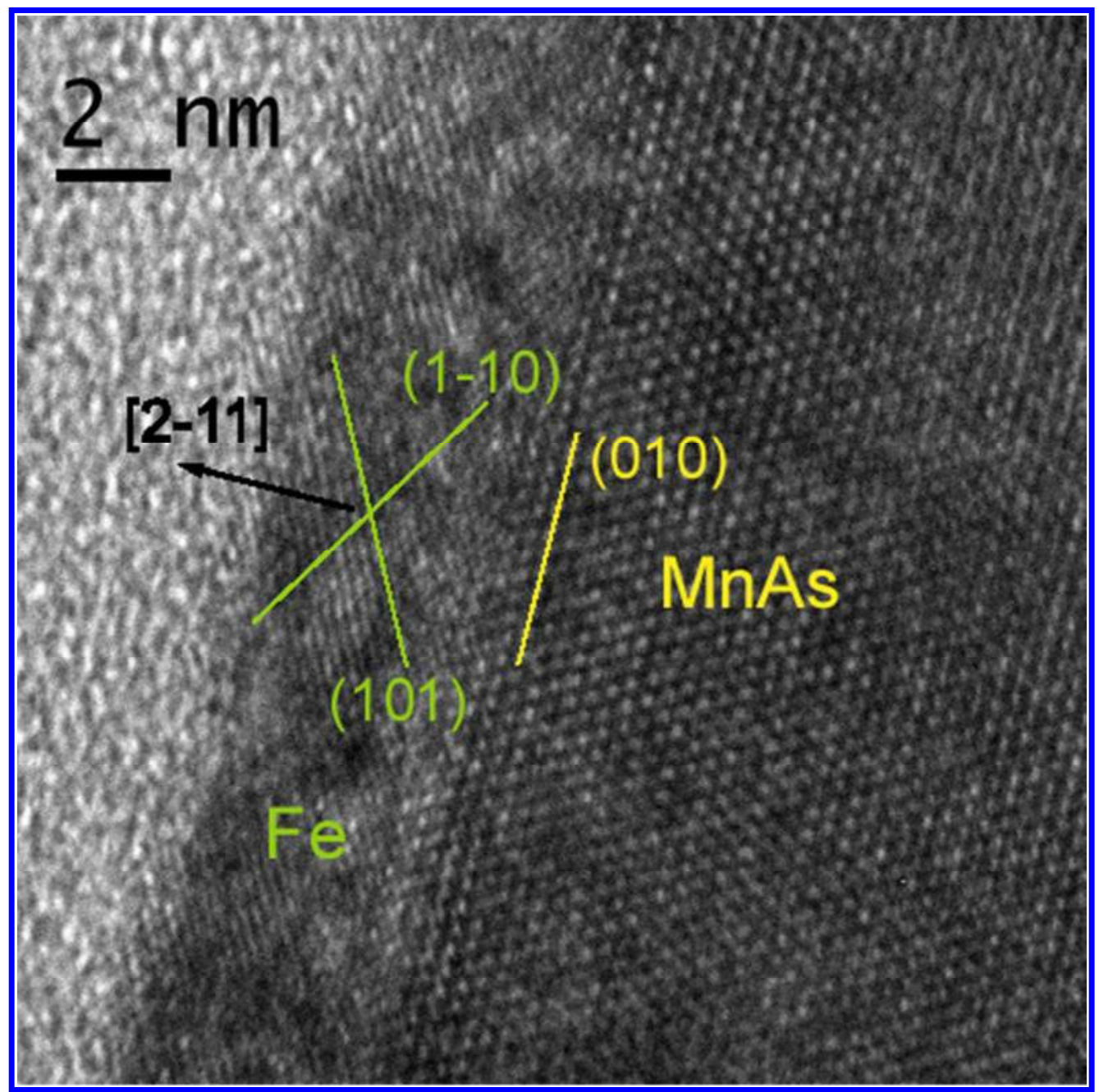

Figure 1. HRTEM cross-sectional image of $\mathrm{Fe} / \mathrm{MnAs} / \mathrm{GaAs}(001)$ along $\mathrm{c}$ axis of $\mathrm{MnAs}$ for a thin Fe layer. $180 \times 180 \mathrm{~mm}(72 \times 72 \mathrm{DPI})$ 


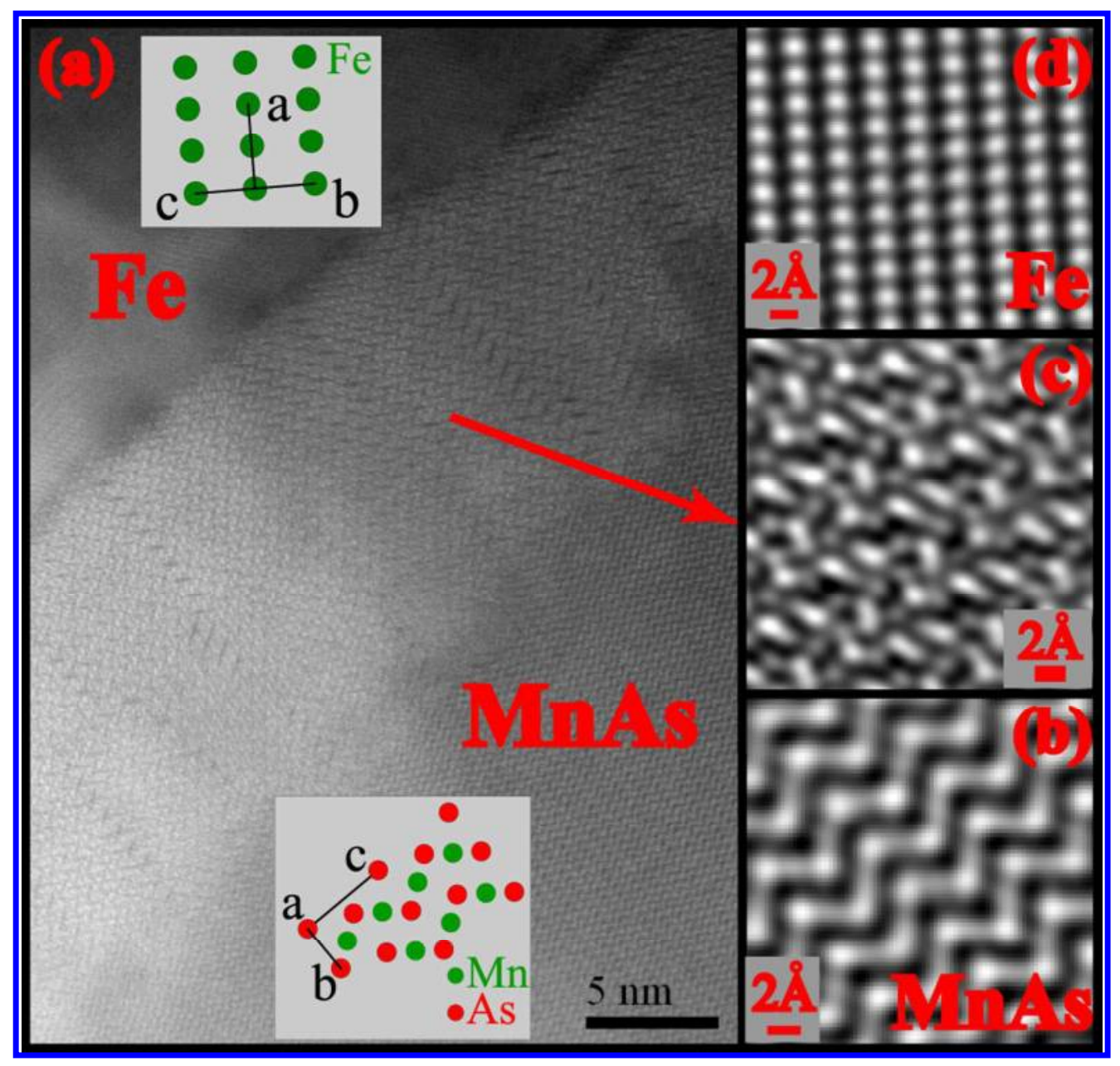

$83 \times 80 \mathrm{~mm}(300 \times 300$ DPI $)$

1
2
3
4
5
6
7
8
9
10
11
12
13
14
15
16
17
18
19
20
21
22
23
24
25
26
27
28
29
30
31
32
33
34
35
36
37
38
39
40
41
42
43
44
45
46
47
48
49
50
51
52
53
54
55
56
57
58
60




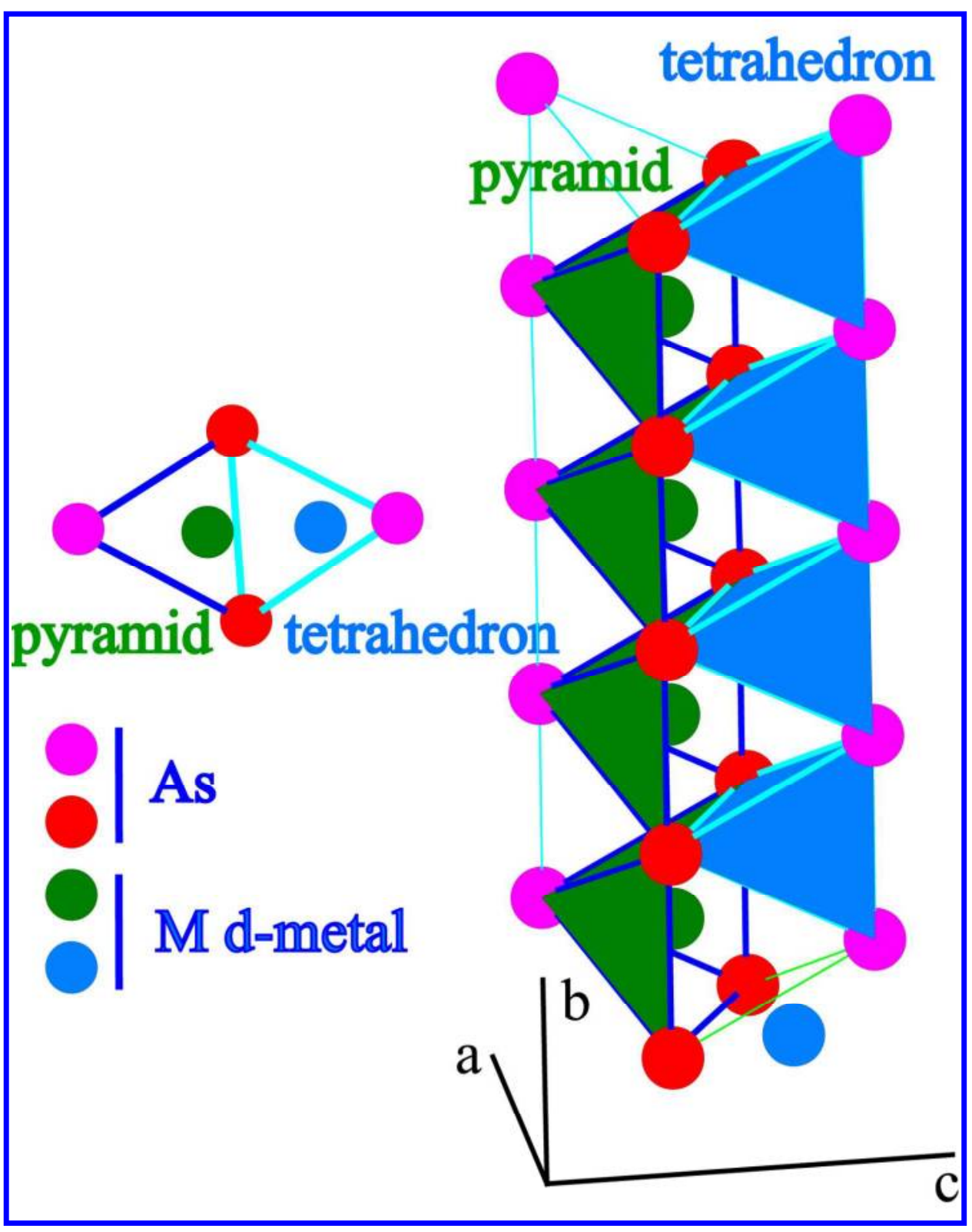

$133 \times 168 \mathrm{~mm}(300 \times 300 \mathrm{DPI})$ 


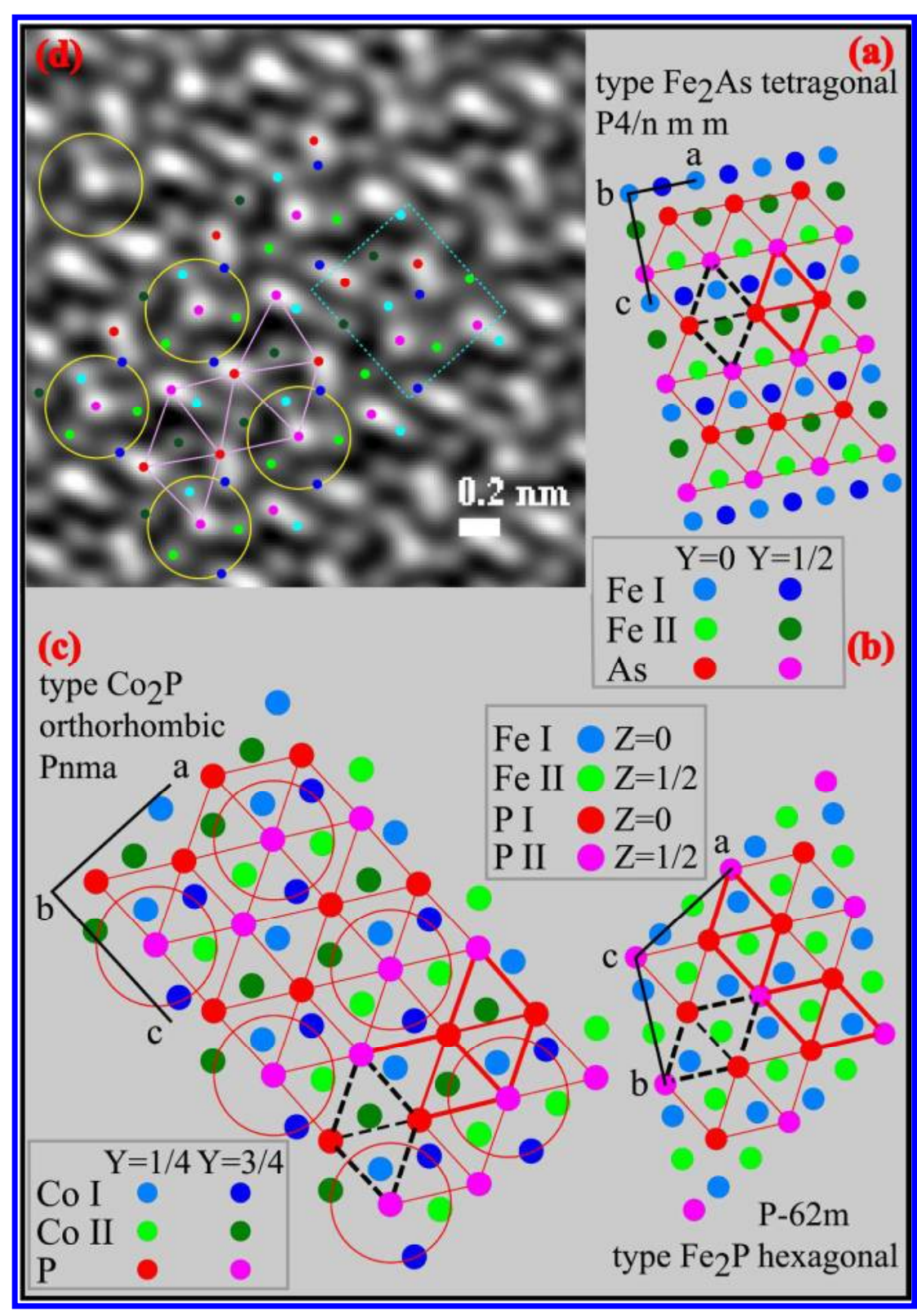

$201 \times 289 \mathrm{~mm}(300 \times 300 \mathrm{DPI})$ 


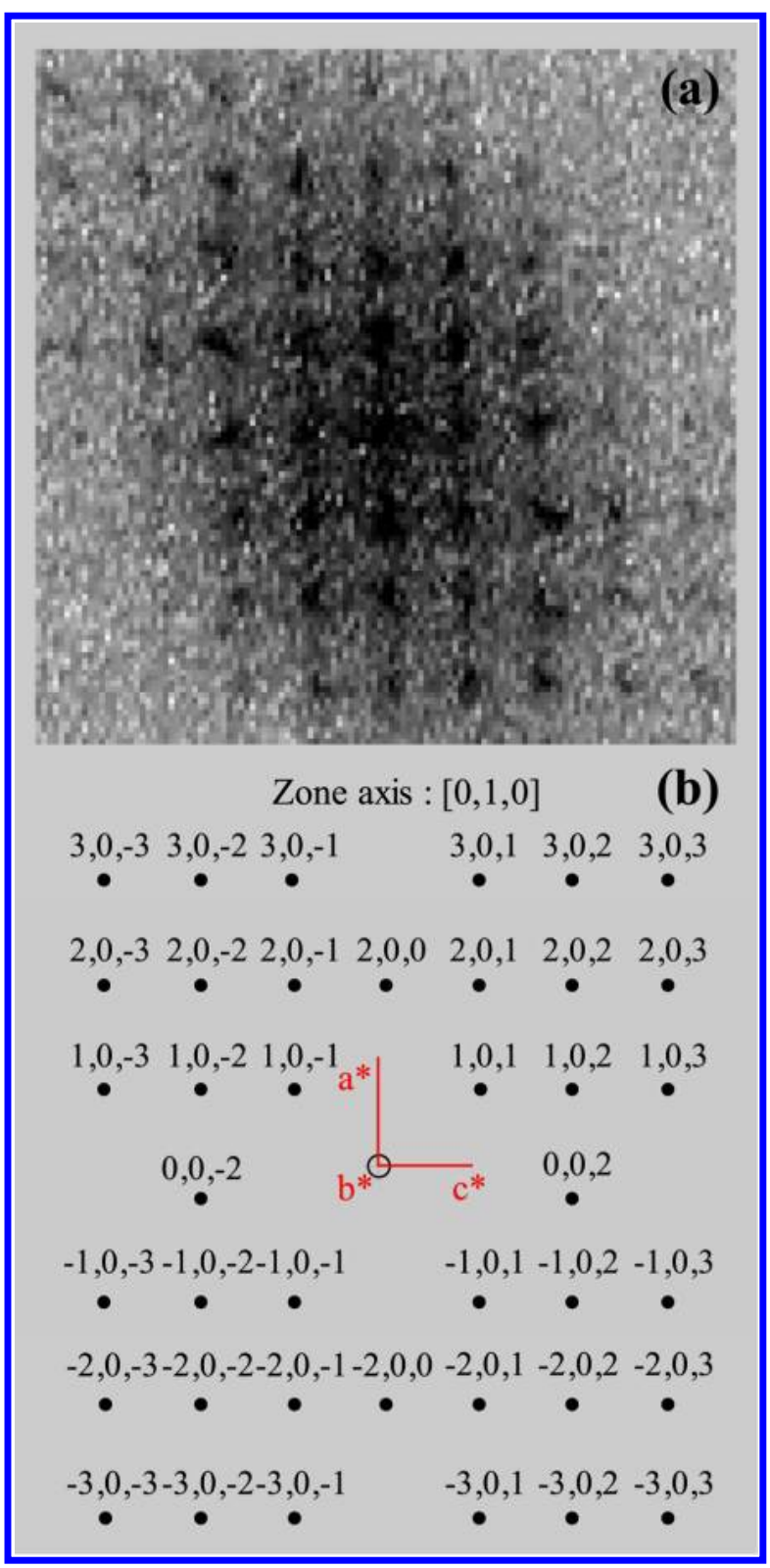

$100 \times 206 \mathrm{~mm}(300 \times 300$ DPI $)$ 


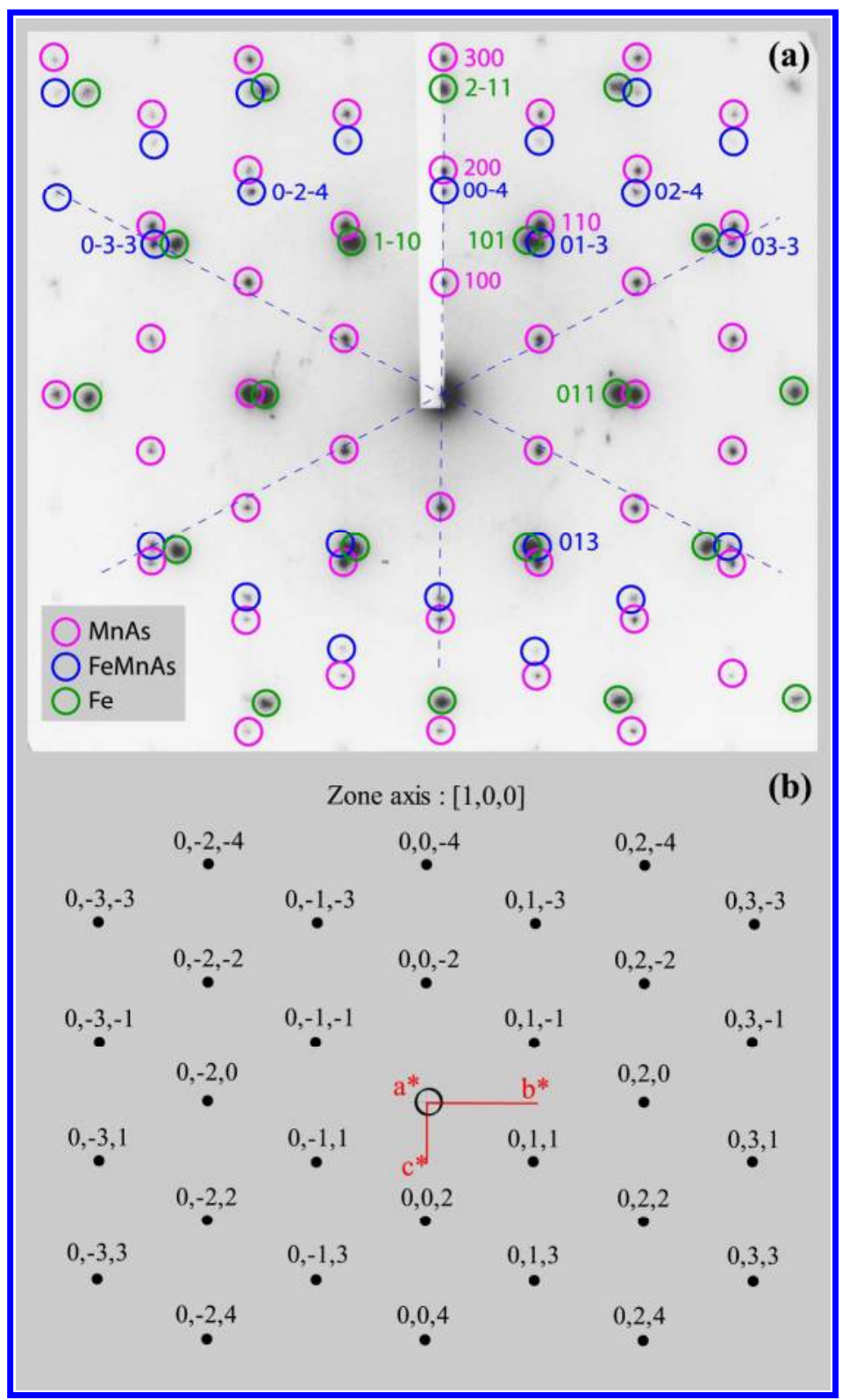

$158 \times 266 \mathrm{~mm}(300 \times 300$ DPI $)$ 


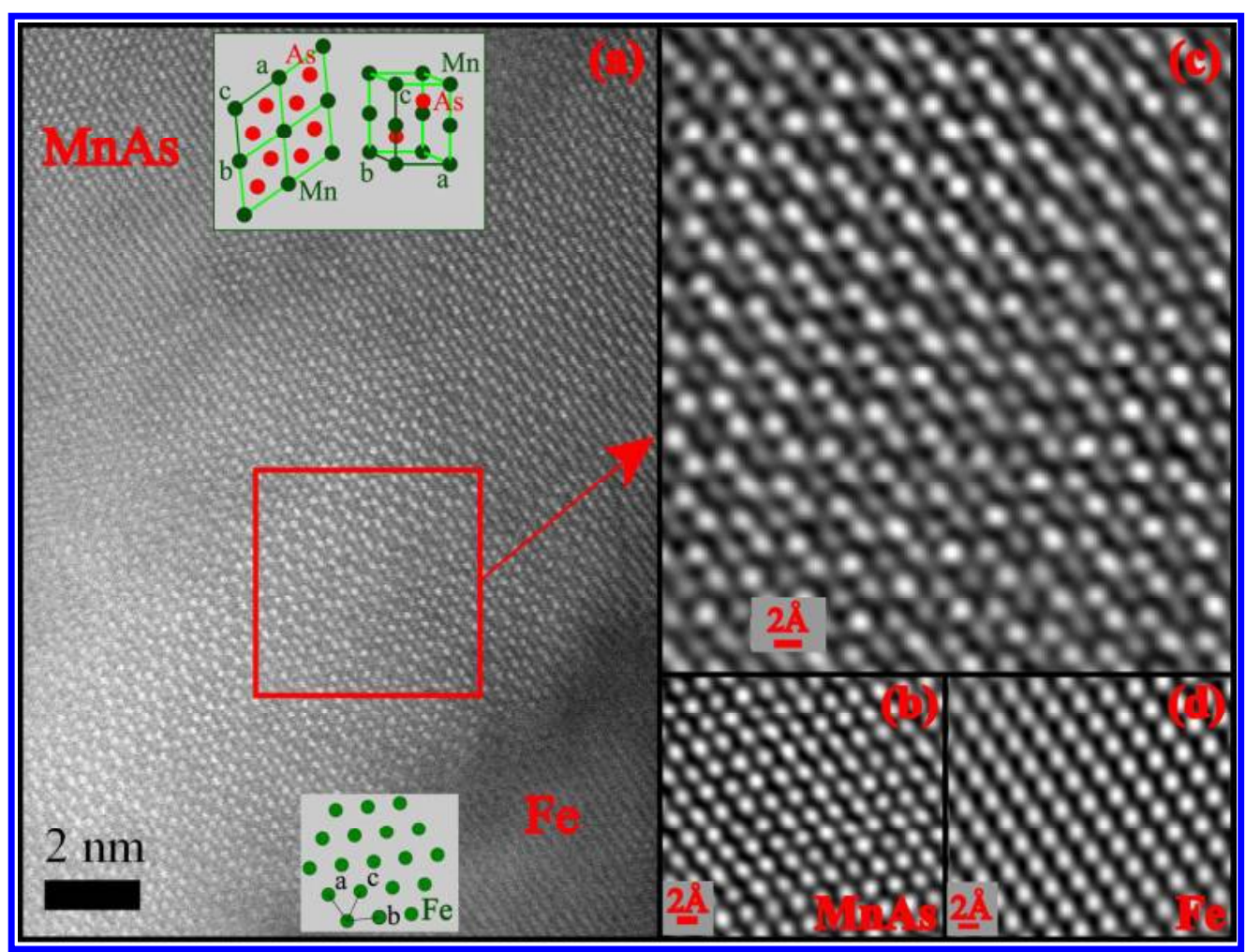

$155 \times 117 \mathrm{~mm}(300 \times 300 \mathrm{DPI})$ 
Figure 8. (a) Filtered HAADF image of the intermediate compound. (b) Schematic structure of orthorhombic Pnma 'FeMnAs' along its [100] zone axis with vacancies (represented by white crosses). Green and blue filled circles correspond respectively to the pyramidal and tetrahedral sites occupied by the metal atoms. White crosses symbolize the vacancies in the structure. $177 \times 102 \mathrm{~mm}(300 \times 300 \mathrm{DPI})$ 